\title{
Which Sagittal Assessment System Can Effectively Predict the Risk of Mechanical Complications in the Treatment of Elderly Patients With Adult Degenerative Scoliosis? Roussouly Classification or Global Alignment and Proportion (GAP) Score
}

\section{Xiangyao Sun}

Xuanwu Hospital Capital Medical University https://orcid.org/0000-0001-9385-2402

Wenzhi Sun

Xuanwu Hospital

\section{Siyuan Sun}

Purdue University

Hailiang Hu

Xuanwu Hospital

Sitao Zhang

Xuanwu Hospital

Chao Kong

Xuanwu Hospital

Shibao Lu (D 15624967325@163.com)

Xuanwu Hospital

\section{Research article}

Keywords: adult degenerative scoliosis, Roussouly classification, Global Alignment and Proportion Score, mechanical complications, effectiveness

Posted Date: June 14th, 2021

DOI: https://doi.org/10.21203/rs.3.rs-576568/v1

License: (c) (1) This work is licensed under a Creative Commons Attribution 4.0 International License.

Read Full License 


\section{Abstract}

Background: In order to achieve the proper sagittal alignment, previous studies have developed different assessment systems for degenerative spinal deformity which could help us in making treatment strategies. The purpose of our study is to evaluate whether Roussouly classification or GAP score is more appropriate in the prediction of mechanical complications in the treatment of ADS.

Methods: The ADS patients who received long segmental fusion in the treatment during the period from December 2016 to December 2018 were evaluated in this study. The basic information of the patients and all radiologic measurements, which were included in GAP score and Roussouly classification, were collected for analysis. Patients were divided into two groups according to occurrence or absence of mechanical complications for comparison. The correlation between evaluation systems and mechanical complications could be analyzed in logistic regression model via stepwise backward elimination based on the Wald statistics. ROC curve was used to determine the predictability of the evaluation systems in the occurrence of mechanical complications and calculate their cut-off value. A two-tailed $P$ value $<0.05$ was statistically significant for all statistical tests.

Results: A total of 80 cases were included in this study. The results of logistic regression showed: GAP score $(P=0.008)$ and GAP categories $(P=0.007)$ were positively correlated with Mechanical complications; Roussouly score was negatively correlated with mechanical complications $(P=0.034)$; GAP score was positively correlated with PJK $(P=0.021)$; Roussouly score was negatively correlated with implant-related complications $(P=0.018)$; GAP categories were correlated with implant loosening $(P=$ 0.023). Results of ROC showed that GAP score was mostly effective in predicting PJK (AUC $=0.863$ ) and PJF $(A U C=0.724)$; GAP categories $(A U C=0.561)$ was more effective than GAP score $(A U C=0.555)$ in predicting implant-related complications.

Conclusions: Roussouly-type matching could not accurately predict the risk of mechanical complications. In contrast, GAP score was mostly effective in predicting PJK and PJF. The GAP score was better than Roussouly classification in predicting mechanical complications.

\section{Introduction}

The three-dimensional deformity occurs in patients with adult degenerative scoliosis (ADS). The coronal correction of frontal deformity was the principle concerned in the past; ADS was found to be deeply affected by the rotational thoracolumbar kyphosis which could alter the sagittal profile [1]. Nowadays, more attention is paid to the sagittal deformity. It was reported that spinal degeneration could decrease lumbar lordosis, increasing thoracic kyphosis, changing the ideal sagittal alignment [2]. In order to achieve the proper sagittal alignment, previous studies have developed different assessment systems for degenerative spinal deformity which could help us in making treatment strategies, such as Scoliosis Research Society (SRS)-Schwab classification [3], Roussouly classification [4] and Global Alignment and Proportion (GAP) Score [5]. 
According to the SRS-Schwab classification $[3,6]$, three targets for corrective surgery realignment were suggested: the pelvic incidence (PI) minus lumbar lordosis (LL) mismatch is less than $10^{\circ}$; pelvic tilt (PT) is less than $20^{\circ}$; sagittal vertical axis (SVA) is less than $4 \mathrm{~cm}$. However, even after matching the targets of Schwab criteria, the mechanical complication rates remain very high [7]. This classification is not effective neither in making the treatment strategy nor in predicting clinical outcome, especially when there is no sagittal malalignment.

In Roussouly classification, 4 types of spinal alignments were described depending on sacral slope (SS) and the shape of LL. This classification was subsequently updated to a modified classification which included a new type, the anteverted type 3 [8]. This new type was characterized by low-grade $\mathrm{PI}, \mathrm{SS}>35^{\circ}$ and low or negative PT [8]. All radiographic factors were compared with ideal spinal alignment to evaluate their deviations from the ideal parameters. In addition, the optimal sagittal alignment was determined on the rate of $\mathrm{PI}$ in proportion to these factors. This is because, $\mathrm{PI}$ is an unchanged parameter [5]. Roussouly classification contribute to the determination of high local stress zones in the whole spine. In this classification, the lower the lumbar lordosis or flat back, the higher the stress is on the disks; the more the lumbar lordosis increased, the more is the contact force on the posterior column [9]. Roussouly classification may help the surgeon predict the best rods bending and the best correction degrees to achieve optimal results. However, degenerative spine modifies the organization of the spinal curves which is responsible for the compensation mechanisms at the spine level or in the pelvis, hips and knees. This can make it difficult to use Roussouly classification in degenerative conditions [10].

Apart from Roussouly classification to help making surgical strategies, GAP score is an alternative that uses PI-based sagittal parameters to quantify the shape and alignment of the sagittal plane. Both Roussouly classification and GAP score share the similar principles to achieve the optimal spinopelvic alignment which includes the restoration of ideal LL, ideal pelvic version, and the ideal lordosis distribution[5]. Planning surgical target in the sagittal plane based on the proportional indices via GAP score can decease the occurrence of mechanical complications[11]. However, no study has compared the effectiveness of these two evaluation systems in predicting mechanical complications after long segmental fusion in the treatment of ADS. Therefore, the purpose of our study is to evaluate whether Roussouly classification or GAP score is more appropriate in the prediction of mechanical complications in the treatment of ADS.

\section{Methods}

\subsection{Inclusion and Exclusion criteria}

The ADS patients who received long segmental fusion in the treatment during the period from December 2016 to December 2018 were evaluated in this study. The basic information of the patients, such as gender, age, BMI (body mass index), follow-up time, blood loss, operation time, vertebrae fused, visual analogue scale (VAS), Japanese Orthopaedic Association (JOA), Oswestry Disability Index (ODI) were collected. The inclusion criteria included: age $>60$ years at the time of attendance; more than 4 vertebral 
levels were fused; coronal Cobb angle $(\mathrm{CA}) \geq 20^{\circ}$, SVA $\geq 5 \mathrm{~cm}, \mathrm{PT} \geq 25^{\circ}$, and thoracic kyphosis $(\mathrm{TK}) \geq 60^{\circ}$; the follow-up time should be more than 2 years. Exclusion criteria included: previous spinal fusion; ADS secondary to syndromic, autoimmune, infectious, tumor, or other pathologic conditions. The written informed consents were signed by all the included cases. The institutional review board approved this study following the declaration of Helsinki principles.

\subsection{Radiographic Measurements and Scoring}

All radiologic measurements included in GAP score and Roussouly classification, such as PI, PT, SS, thoracolumbar kyphosis (TLK), TK, LL, L4-S1 lordosis, global tilt (GT), SVA, the number of vertebrae included in the lordosis (NVL), the lumbar sagittal apex (LA) and the inflexion point (IP) were recorded at 6 weeks postoperatively (Supplementary file 1). All radiographs were analyzed by validated software (Surgimap, Nemaris Inc., New York, NY). All data were measured separately by two researchers. When discrepancies arose, a consensus would be taken by the coauthors after discussion.

All Roussouly types have specific lumbar sagittal apexes, level of inflexion points, and NVLs [4, 8, 12, 13]. Standard values of these parameters for Roussouly types are shown in Supplementary file 2, as summarized previously [1]. It has been demonstrated that over-correction (a shape resembling a "higher" Roussouly type than ideal shape) in the realignment of ADS was worse than under-correction (realign the spine in a shape resembling a "lower" profile than ideal shape) or ideal realignment in the treatment [1]. Roussouly modifiers of ADS patients were defined in this study. The definition was as follows: modifier " 0 ", patients with ideal profiles; modifier "+", patients with under-corrected profiles; modifier " ++ ", patients with over-corrected profiles. The Roussouly modifiers were then statistically weighted ( 1 for modifier " 0 ", 2 for modifier " + " and 3 for modifier "++").

The GAP score ranges from 0 to 13 points. It included RPV (relative pelvic version), RLL (relative lumbar lordosis), LDI (lordosis distribution index), RSA (relative spinopelvic alignment) and age [5]. The cut-off values of GAP score are as follows: a GAP score of 0 to 2 indicates a proportioned spinopelvic position; a GAP score of 3 to 6 is defined as moderately disproportioned; a GAP score more than 6 is defined as severely disproportioned (Supplementary file 3) [5].

\subsection{Mechanical Complications}

The mechanical complications discussed in this study included: proximal junctional kyphosis/ failure (PJK or PJF), distal junctional kyphosis/ failure (DJK or DJF), and implant-related complications [5]. PJK was defined as the kyphosis between UIV (upper instrumented vertebra) and UIV +2 increased $\geq 10^{\circ}$ in between early postoperative and follow-up radiographs. PJF was the fracture of UIV or UIV +1 , pullout of instrumentation at UIV, and/or sagittal subluxation. DJK or DJF was the postoperative kyphosis angle between LIV (lower instrumented vertebra) and LIV-1 increased $\geq 10^{\circ}$, and/or pullout of instrumentation at LIV. Implant-related complications were other radiographic implant-related complications such as implant loosening, implant breakage or implant pullout. Patients were divided into two groups according to occurrence or absence of mechanical complications for comparison. 


\subsection{Statistical Analysis}

The statistical analysis was performed using the SPSS 17.0 (SPSS Inc, Richmond, CA, USA). Continuous variables were reported as mean \pm standard deviations. Kolmogorov-Smirnov test was performed to the normal distribution of the data. Normally distributed values were analyzed with independent Student $t$ test. Skewed values were analyzed with Kruskal-Wallist test. Categorical variables were reported as the number of cases and compared using Pearson's Chi-square test. The correlation between evaluation systems and mechanical complications could be found by odds ratio (OR) and $95 \%$ confidence interval (Cl) in logistic regression model via stepwise backward elimination based on the Wald statistics. ROC (receiver operating characteristic) curve was used to determine the predictability of the evaluation systems in the occurrence of mechanical complications and calculate their cut-off value. A two-tailed $P$ value $<0.05$ was statistically significant for all statistical tests.

\section{Results}

\subsection{Demographics}

A total of 80 cases were included in this study (Table 1 ). The mean age is $76.5 \pm 2.5$ years old. The mean follow-up is $19.3 \pm 6.2$ months. Implant-related complication (42.5\%) has the highest incidence in mechanical complications (51.3\%). The most common implant-related complication is implant loosening (37.5\%). The postoperative radiographic parameters and clinical scoring systems were significantly improved compared with the preoperative data (Table 2). 
Table 1

Characteristics of the included cases

\begin{tabular}{|ll|}
\hline Variables & Data \\
\hline Cases & 80 \\
\hline Female (n, \%) & $65(81.3 \%)$ \\
\hline Age (years) & $76.5 \pm 2.5$ \\
\hline BMI & $26.8 \pm 3.8$ \\
\hline Follow-up time (months) & $19.3 \pm 6.2$ \\
\hline Blood loss (ml) & $1052.2 \pm 330.0$ \\
\hline Operation time (min) & $450.9 \pm 141.4$ \\
\hline Vertebrae fused (n) & $6.0 \pm 1.9$ \\
\hline Mechanical complications (n, \%) & $41(51.3 \%)$ \\
\hline PJK (n, \%) & $5(6.25 \%)$ \\
\hline PJF (n, \%) & $2(2.5 \%)$ \\
\hline DJK or DJF (n, \%) & $2(2.5 \%)$ \\
\hline Implant-related complications (n, \%) & $34(42.5 \%)$ \\
\hline Implant loosening (n, \%) & $30(37.5 \%)$ \\
\hline Implant breakage (n, \%) & $4(5 \%)$ \\
\hline $\begin{array}{l}\text { Notice: Pre, preoperative; Post, postoperative; BMI, body mass index; PJK, proximal junctional } \\
\text { kyphosis; PJF, proximal junctional failure; DJK, distal junctional kyphosis; DJF, distal junctional }\end{array}$ & \\
\hline failure. & \\
\hline
\end{tabular}


Table 2

Radiographic parameters

\begin{tabular}{|llll|}
\hline Items & Pre-data & Post-data & P value \\
\hline $\mathrm{CA}\left(^{\circ}\right)$ & $22.1 \pm 6.9$ & $7.4 \pm 2.3$ & $<0.001$ \\
\hline TK $\left(^{\circ}\right)$ & $46.2 \pm 30.2$ & $30.8 \pm 20.1$ & $<0.001$ \\
\hline LL $\left(^{\circ}\right)$ & $25.0 \pm 14.6$ & $33.9 \pm 10.7$ & $<0.001$ \\
\hline SS $\left(^{\circ}\right)$ & $24.8 \pm 9.5$ & $28.2 \pm 7.2$ & 0.024 \\
\hline PT $\left(^{\circ}\right)$ & $26.1 \pm 14.5$ & $22.3 \pm 9.9$ & 0.010 \\
\hline SVA $(\mathrm{cm})$ & $9.6 \pm 3.7$ & $3.6 \pm 3.4$ & $<0.001$ \\
\hline VAS & $6.5 \pm 1.7$ & $2.7 \pm 0.8$ & $<0.001$ \\
\hline JOA score & $3.8 \pm 1.1$ & $6.1 \pm 1.8$ & $<0.001$ \\
\hline ODI & $60.0 \pm 24.3$ & $26.9 \pm 12.8$ & $<0.001$ \\
\hline $\begin{array}{l}\text { Notice: Pre, preoperative; Post, postoperative; CA, coronal Cobb angle; TK, thoracal kyphosis; LL, } \\
\text { lumbar lordosis; SS, sacral slope; PT, pelvic tilt; SVA, sagittal vertical axis; VAS, visual analogue scale; } \\
\text { JOA, Japanese Orthopaedic Association; ODI, Oswestry Disability Index. }\end{array}$ \\
\hline
\end{tabular}

\subsection{Comparison of parameters in Roussouly classification}

More cases without mechanical complications were Roussouly-type 1 compared to those with mechanical complications $(P=0.035)$. Compared to cases with mechanical complications, there was more patients without mechanical complications matching ideal LA $(P<0.001)$. There were more patients who matched Roussouly-type in no mechanical complication group compared with that in mechanical complication groups $(P=0.048)$. The Roussouly score in no mechanical complication group was more than that in mechanical group $(P=0.032)($ Table 3$)$. 
Table 3

Comparison of parameters in Roussouly classification between groups with or without mechanical complications

\begin{tabular}{|c|c|c|c|}
\hline Variables & $\begin{array}{l}\text { Mechanical complication } \\
(n=41)\end{array}$ & $\begin{array}{l}\text { No mechanical complication } \\
(n=39)\end{array}$ & $P$ value \\
\hline Roussouly-type & & & 0.082 \\
\hline 1 & $4(9.8 \%)$ & $11(28.2 \%)$ & 0.035 \\
\hline 2 & $5(12.2 \%)$ & $6(15.4 \%)$ & 0.679 \\
\hline 3 & 23(56.1\%) & $19(48.7 \%)$ & 0.509 \\
\hline 4 & $9(22.0 \%)$ & $3(7.7 \%)$ & 0.074 \\
\hline Post-LA & & & 0.262 \\
\hline L2 & $2(4.9 \%)$ & $0(0 \%)$ & 0.162 \\
\hline L2/3 & $3(7.3 \%)$ & $0(0 \%)$ & 0.085 \\
\hline L3 & $4(9.8 \%)$ & $6(15.4 \%)$ & 0.447 \\
\hline L3/4 & $3(7.3 \%)$ & $3(7.7 \%)$ & 0.949 \\
\hline L4 & 12(29.3\%) & $16(41.0 \%)$ & 0.270 \\
\hline L4/5 & $11(26.8 \%)$ & $6(15.4 \%)$ & 0.211 \\
\hline L5 & $6(14.6 \%)$ & $8(20.5 \%)$ & 0.489 \\
\hline Ideal LA & & & 0.082 \\
\hline L3/4 & $9(30.0 \%)$ & $3(7.7 \%)$ & 0.074 \\
\hline L4 & $23(56.1 \%)$ & 19(48.7\%) & 0.509 \\
\hline L4/5 & $5(12.2 \%)$ & $6(15.4 \%)$ & 0.679 \\
\hline L5 & $4(9.8 \%)$ & $11(28.2 \%)$ & 0.035 \\
\hline Match ideal LA & $9(30.0 \%)$ & $25(64.1 \%)$ & $<0.001$ \\
\hline Post-IP & & & 0.033 \\
\hline T11 & $1(2.4 \%)$ & $0(0 \%)$ & 0.326 \\
\hline T12 & $8(19.5 \%)$ & $2(5.1 \%)$ & 0.053 \\
\hline L1 & 19(46.3\%) & $15(38.5 \%)$ & 0.476 \\
\hline $\mathrm{L} 1 / 2$ & $0(0 \%)$ & $2(5.1 \%)$ & 0.142 \\
\hline L2 & $8(19.5 \%)$ & 18(46.2\%) & 0.011 \\
\hline
\end{tabular}




\begin{tabular}{|llll|}
\hline Variables & $\begin{array}{l}\text { Mechanical complication } \\
(\mathbf{n = 4 1 )}\end{array}$ & $\begin{array}{l}\text { No mechanical complication } \\
(\mathbf{n}=39)\end{array}$ & P value \\
\hline L3 & $5(12.2 \%)$ & $2(5.1 \%)$ & 0.264 \\
\hline Ideal IP & & $3(7.7 \%)$ & 0.082 \\
\hline T12 & $9(22.0 \%)$ & $19(48.7 \%)$ & 0.074 \\
\hline L1 & $23(56.1 \%)$ & $6(15.4 \%)$ & 0.509 \\
\hline L2 & $5(12.2 \%)$ & $11(28.2 \%)$ & 0.679 \\
\hline L3 & $4(9.8 \%)$ & $11(28.2 \%)$ & 0.035 \\
\hline Match ideal Post-IP & $15(36.6 \%)$ & $48.7 \pm 8.9$ & 0.424 \\
\hline Post-PI & $53.1 \pm 13.0$ & $19.4 \pm 5.3$ & 0.082 \\
\hline Post-PT & $25.0 \pm 12.2$ & $28.6 \pm 8.3$ & 0.009 \\
\hline Post-SS & $27.7 \pm 6.0$ & $9(23.1 \%)$ & 0.577 \\
\hline Match Roussouly-type & $3(7.3 \%)$ & $0.9 \pm 0.7$ & 0.048 \\
\hline Roussouly score & $0.6 \pm 0.6$ & & 0.032 \\
\hline $\begin{array}{l}\text { Notice: Post, postoperative; LA, lumbar apex; IP, inflexion point; PI, pelvic incidence; PT, pelvic tilt; SS, } \\
\text { sacral slope }\end{array}$ & & \\
\hline
\end{tabular}

\subsection{Comparison of parameters in GAP score}

The GAP score in mechanical group was higher than that in no mechanical complication $(P=0.005)$. The Post-RPV score $(P=0.003)$ and Post-GT $(P=0.007)$ in mechanical group were significantly higher than those in no mechanical group. The Post-RPV $(P=0.019)$ and Post- $R L L(P=0.006)$ in mechanical group were significantly lower than those in no mechanical group. The number of patients with moderately disproportioned GAP score in no mechanical group was more than that in mechanical group $(P=0.010)$. There were more patients with severely disproportioned GAP score in mechanical group compared with those in no mechanical group $(P=0.003)$ (Table 4$)$. 
Table 4

Comparison of parameters in GAP score between groups with or without mechanical complications

\begin{tabular}{|llll|}
\hline Post-variables & $\begin{array}{l}\text { Mechanical complication } \\
(\mathbf{n}=\mathbf{4 1})\end{array}$ & $\begin{array}{l}\text { No mechanical complication } \\
(\mathbf{n}=39)\end{array}$ & P value \\
\hline GAP score & $8.8 \pm 2.7$ & $6.8 \pm 3.4$ & 0.005 \\
\hline Post-PI & $53.1 \pm 13.0$ & $48.7 \pm 8.9$ & 0.082 \\
\hline Post-SS & $27.7 \pm 6.0$ & $28.6 \pm 8.3$ & 0.577 \\
\hline Ideal SS & $40.3 \pm 7.7$ & $37.7 \pm 5.2$ & 0.082 \\
\hline Post-RPV & $-12.6 \pm 7.7$ & $-9.1 \pm 5.1$ & 0.019 \\
\hline Post-RPV score & $2.0 \pm 1.0$ & $1.3 \pm 1.1$ & 0.003 \\
\hline Post-LL & $32.3 \pm 11.0$ & $35.5 \pm 10.2$ & 0.177 \\
\hline Ideal LL & $61.9 \pm 8.1$ & $59.2 \pm 5.5$ & 0.082 \\
\hline Post- RLL & $-29.7 \pm 10.6$ & $-23.7 \pm 8.0$ & 0.006 \\
\hline Post-RLL score & $2.5 \pm 0.7$ & $2.2 \pm 0.9$ & 0.054 \\
\hline Post-LDI & $0.9 \pm 0.3$ & $0.8 \pm 0.2$ & 0.200 \\
\hline Post-LDI score & $1.7 \pm 1.5$ & $1.3 \pm 1.5$ & 0.256 \\
\hline Post-GT & $27.3 \pm 13.5$ & $20.6 \pm 6.6$ & 0.007 \\
\hline Post-Age & $76.1 \pm 2.3$ & $76.9 \pm 2.7$ & 0.003 \\
\hline GAP score categories & & & 0.012 \\
\hline Proportioned & $1(2.4 \%)$ & $3(7.7 \%)$ & 0.281 \\
\hline Moderately disproportioned & $7(17.1 \%)$ & $17(43.6 \%)$ & 0.010 \\
\hline Severely disproportioned & $33(80.5 \%)$ & $19(48.7 \%)$ & \\
\hline GAP score, global alignment and proportion score; Post, postoperative; SS, sacral slope; LL, lumbar \\
\hline lordosis; RPV, relative pelvic version; RLL, relative lumbar lordosis; LDI, lordosis distribution index; GT, \\
\hline
\end{tabular}

\subsection{Correlations between evaluation systems and mechanical complications}

The results of logistic regression showed: GAP score $(P=0.008)$ and GAP categories $(P=0.007)$ were positively correlated with Mechanical complications; Roussouly score was negatively correlated with mechanical complications $(P=0.034)$; GAP score was positively correlated with PJK $(P=0.021)$; 
Roussouly score was negatively correlated with implant-related complications $(P=0.018)$; GAP categories were correlated with implant loosening $(P=0.023)$ (Table 5). 
Table 5

Correlations between evaluation systems and mechanical complications in logistic regression

\begin{tabular}{|c|c|c|c|c|c|c|}
\hline Characteristics & $\begin{array}{l}\text { B } \\
\text { value }\end{array}$ & SE & $\begin{array}{l}\text { Wald } \\
\text { value }\end{array}$ & $\begin{array}{l}\mathrm{P} \\
\text { value }\end{array}$ & $\begin{array}{l}\text { Exp (B) } \\
\text { value }\end{array}$ & $95 \% \mathrm{Cl}$ \\
\hline \multicolumn{7}{|l|}{ Mechanical complications } \\
\hline GAP score & 1.602 & 0.079 & 7.103 & 0.008 & 1.233 & $\begin{array}{l}(1.057, \\
1.439)\end{array}$ \\
\hline Contant & -1.602 & 0.667 & 5.770 & 0.016 & 0.201 & \\
\hline GAP catergories & 1.211 & 0.449 & 7.283 & 0.007 & 3.358 & $\begin{array}{l}(1.393, \\
8.092)\end{array}$ \\
\hline Contant & -1.910 & 0.779 & 6.017 & 0.014 & 0.148 & \\
\hline Roussouly score & -0.721 & 0.341 & 4.481 & 0.034 & 0.486 & $\begin{array}{l}(0.249, \\
0.948)\end{array}$ \\
\hline Contant & 0.590 & 0.342 & 2.969 & 0.085 & 1.804 & \\
\hline Match Roussouly-type & -0.668 & 0.355 & 3.536 & 0.060 & 0.513 & $\begin{array}{l}(0.256, \\
1.029)\end{array}$ \\
\hline Contant & 0.236 & 0.244 & 0.937 & 0.333 & 1.267 & \\
\hline \multicolumn{7}{|l|}{ PJK } \\
\hline GAP score & 0.656 & 0.283 & 5.362 & 0.021 & 1.927 & $\begin{array}{l}(1.106, \\
3.357)\end{array}$ \\
\hline Contant & -9.199 & 3.195 & 8.287 & 0.004 & $<0.001$ & \\
\hline Roussouly score & 0.108 & 0.654 & 0.027 & 0.869 & 1.114 & $\begin{array}{l}(0.309, \\
4.016)\end{array}$ \\
\hline Contant & -2.792 & 0.696 & 16.071 & $\begin{array}{l}< \\
0.001\end{array}$ & 0.061 & \\
\hline \multicolumn{7}{|l|}{ PJF } \\
\hline GAP score & 0.269 & 0.291 & 0.854 & 0.355 & 1.308 & $\begin{array}{l}(0.740, \\
2.313)\end{array}$ \\
\hline Contant & -6.081 & 2.981 & 4.161 & 0.041 & 0.002 & \\
\hline \multicolumn{7}{|l|}{ DJK or DJF } \\
\hline GAP score & -0.177 & 0.218 & 0.659 & 0.417 & 0.838 & $\begin{array}{l}(0.547, \\
1.284)\end{array}$ \\
\hline Contant & -2.431 & 1.504 & 2.614 & 0.106 & 0.088 & \\
\hline GAP catergories & -1.349 & 1.003 & 1.808 & 0.179 & 0.259 & $\begin{array}{l}(0.036, \\
1.854)\end{array}$ \\
\hline
\end{tabular}




\begin{tabular}{|c|c|c|c|c|c|c|}
\hline Characteristics & $\begin{array}{l}\text { B } \\
\text { value }\end{array}$ & SE & $\begin{array}{l}\text { Wald } \\
\text { value }\end{array}$ & $\begin{array}{l}\mathrm{P} \\
\text { value }\end{array}$ & $\begin{array}{l}\text { Exp (B) } \\
\text { value }\end{array}$ & $95 \% \mathrm{Cl}$ \\
\hline Contant & -1.865 & 1.269 & 2.169 & 0.141 & 0.155 & \\
\hline Roussouly score & -0.581 & 1.150 & 0.255 & 0.613 & 0.559 & $\begin{array}{l}(0.059) \\
5.328)\end{array}$ \\
\hline Contant & -3.301 & 0.923 & 12.787 & ${ }_{0.001}^{<}$ & 0.037 & \\
\hline \multicolumn{7}{|l|}{$\begin{array}{l}\text { Implant-related } \\
\text { complications }\end{array}$} \\
\hline GAP score & 0.085 & 0.073 & 1.372 & 0.241 & 1.089 & $\begin{array}{l}(0.944, \\
1.258)\end{array}$ \\
\hline Contant & -0.979 & 0.627 & 2.443 & 0.118 & 0.376 & \\
\hline GAP catergories & 0.573 & 0.416 & 1.897 & 0.168 & 1.774 & $\begin{array}{l}(0.785, \\
4.010)\end{array}$ \\
\hline Contant & -1.231 & 0.721 & 2.910 & 0.088 & 0.292 & \\
\hline Roussouly score & -0.846 & 0.358 & 5.588 & 0.018 & 0.429 & $\begin{array}{l}(0.213, \\
0.865)\end{array}$ \\
\hline Contant & 0.301 & 0.337 & 0.801 & 0.371 & 1.352 & \\
\hline Match Roussouly-type & -0.922 & 0.710 & 1.686 & 0.194 & 0.398 & $\begin{array}{l}(0.099, \\
1.5999)\end{array}$ \\
\hline Contant & -0.177 & 0.243 & 0.528 & 0.467 & 0.838 & \\
\hline \multicolumn{7}{|l|}{ Implant loosening } \\
\hline GAP score & 0.151 & 0.079 & 3.682 & 0.055 & 1.163 & $\begin{array}{l}(0.997, \\
1.357)\end{array}$ \\
\hline Contant & -1.730 & 0.694 & 6.219 & 0.013 & 0.177 & \\
\hline GAP catergories & 1.127 & 0.495 & 5.180 & 0.023 & 3.087 & $\begin{array}{l}(1.169, \\
8.147)\end{array}$ \\
\hline Contant & -2.382 & 0.888 & 7.199 & 0.007 & 0.092 & \\
\hline Roussouly score & -0.511 & 0.347 & 2.169 & 0.141 & 0.600 & $\begin{array}{l}(0.304, \\
1.184)\end{array}$ \\
\hline Contant & -0.144 & 0.333 & 0.187 & 0.665 & 0.866 & \\
\hline Match Roussouly-type & -0.681 & 0.711 & 0.916 & 0.338 & 0.506 & $\begin{array}{l}(0.126, \\
2.040)\end{array}$ \\
\hline Contant & -0.418 & 0.248 & 2.841 & 0.092 & 0.659 & \\
\hline
\end{tabular}




\begin{tabular}{|c|c|c|c|c|c|c|}
\hline Characteristics & $\begin{array}{l}\text { B } \\
\text { value }\end{array}$ & SE & $\begin{array}{l}\text { Wald } \\
\text { value }\end{array}$ & $\begin{array}{l}P \\
\text { value }\end{array}$ & $\begin{array}{l}\text { Exp (B) } \\
\text { value }\end{array}$ & $95 \% \mathrm{Cl}$ \\
\hline GAP score & -0.260 & 0.165 & 2.496 & 0.114 & 0.771 & $\begin{array}{l}(0.558, \\
1.065)\end{array}$ \\
\hline Contant & -1.219 & 1.037 & 1.380 & 0.240 & 0.296 & \\
\hline GAP catergories & -1.425 & 0.748 & 3.628 & 0.057 & 0.241 & $\begin{array}{l}(0.056, \\
1.042)\end{array}$ \\
\hline Contant & -1.031 & 0.961 & 1.150 & 0.284 & 0.357 & \\
\hline
\end{tabular}

\subsection{ROC of evaluation systems in predicting mechanical complications}

Results of ROC showed that GAP score was more effective in prediction mechanical complications than Roussouly classification (Fig. 1). GAP score (Cut-off value $=10$ ) was mostly effective in predicting PJK $(A U C=0.863)$ and PJF (AUC $=0.724)$. GAP categories $(A U C=0.561$, Cut-off value $=$ Severely disproportioned) was more effective than GAP score $(A U C=0.555$, Cut-off value $=5)$ in predicting implant-related complications; In other respects, however, the GAP score is superior to the GAP categories (Table 6). 
Table 6

Results of ROC analyzing evaluation systems in predicting mechanical complications

$\begin{array}{lllll}\text { Characteristics } & \text { AUC } & \text { Cut-off value } & \text { Sensitivity } & \begin{array}{l}1- \\ \text { Specificity index }\end{array}\end{array}$

Mechanical

complications

$\begin{array}{llllll}\text { GAP score } & 0.669 & 8 & 0.805 & 0.385 & 0.420 \\ \text { GAP catergories } & 0.660 & \begin{array}{l}\text { Moderately } \\ \text { disproportioned }\end{array} & 0.976 & 0.923 & 0.053\end{array}$

PJK

$\begin{array}{llllll}\text { GAP score } & 0.863 & 10 & 1.000 & 0.373 & 0.627 \\ \text { GAP catergories } & 0.687 & \begin{array}{l}\text { Severely } \\ \text { disproportioned }\end{array} & 1.000 & 0.627 & 0.373\end{array}$

$\begin{array}{llllll}\text { Roussouly score } & 0.543 & 1 & 0.800 & 0.587 & 0.213\end{array}$

PJF

$\begin{array}{llllll}\text { GAP score } & 0.724 & 10 & 1.000 & 0.397 & 0.603 \\ \text { GAP catergories } & 0.679 & \begin{array}{l}\text { Severely } \\ \text { disproportioned }\end{array} & 1.000 & 0.641 & 0.359\end{array}$

DJK or DJF

GAP score

$0.442 \quad 11$

0.500

0.141

0.359

Implant-related

complications

$\begin{array}{llllll}\text { GAP score } & 0.555 & 5 & 0.912 & 0.696 & 0.216 \\ \text { GAP catergories } & 0.561 & \begin{array}{l}\text { Severely } \\ \text { disproportioned }\end{array} & 0.706 & 0.609 & 0.097\end{array}$

Implant loosening

$\begin{array}{llllll}\text { GAP score } & 0.615 & 8 & 0.800 & 0.480 & 0.320 \\ \text { GAP catergories } & 0.628 & \begin{array}{l}\text { Severely } \\ \text { disproportioned }\end{array} & 0.800 & 0.560 & 0.24\end{array}$

Implant breakage
GAP score
$0.217 \quad 8$
1.000
0.947
0.053

Notice: ROC, receiver operator characteristic curve; AUC, area under the curve; GAP score, global alignment and proportion score; PJK, proximal junctional kyphosis; PJF, proximal junctional failure; DJK, distal junctional kyphosis; DJF, distal junctional failure. 


\section{Discussion}

Previous studies showed that the postoperative complication rates $(8.4-42 \%)$, revision rates $(9-17.6 \%)$ in ADS were still high, which could increase after long-term follow-up [14, 15]. Increased junctional stress concentration ADS to the collapse of the implant or vertebra; this could cause mechanical complications such as PJK, distal junction kyphosis (DJK), pseudoarthrosis, rod breakage or vertebral fracture $[5,9,11$, 16]. The patients included in this study were all elderly, and therefore had poor tolerance to spinal stress concentration. The most common mechanical complication in this study was screw loosening. This is due to a decrease in bone density in older patients. Therefore, the stress concentration on the contact surface between screw and bone structure can be alleviated by screw loosening [16].

Several parameters are reported to be associated with mechanical complications, including TK, SVA, and SS [16-19]. The PI-LL in SRS-Schwab classification could be used to quantify spinopelvic harmony, which is also thought to be an important vital factor for predicting mechanical complication and is usually used to predict better postoperative health-related quality of life [20, 21]. However, this kind of classification is based on linear absolute numerical parameters, which could not include the whole spectrum of PI. The realignment targets in SRS-Schwab classification could be misleading when the PI values were near the ends of normality (too high or too low) [12]. Therefore, previous studies used the degree of compensation and present PI to estimate the ideal spinal alignment according to Roussoulytypes [22, 23].

Roussouly defined four basic shapes of normal sagittal spine alignment in healthy population based on SS [4]. However, lumbar degeneration and thoracolumbar coronal deformity could modify lumbar lordosis, which could consequently influence SS [2]. Therefore, SS becomes an inadequate parameter to classify sagittal types in pathologic patients. In addition, the Roussouly classification relies on PI which is considered not to vary with age, pathology, or compensation [24]. However, Roussouly classification is mainly based on the classification of normal spine; most of the studies related to the compensatory mechanism of spinal degeneration were cross-sectional studies [12, 22, 25]. In this study, more cases without mechanical complications were Roussouly-type 1 compared to those with mechanical complications. This is because Roussouly-type 1 is a combination of long kyphosis and short lordosis at the lower arc of the spine; the inflexion point, which always represent the region with highest junctional stress concentration, has already been fixed in the central structure of the long-segment internal fixation system[26]. Our study showed: there were more patients who matched Roussouly-type in no mechanical complication group compared with that in mechanical complication groups; compared to cases with mechanical complications, there was more patients without mechanical complications matching ideal LA. These results suggested that the difference in Roussouly type matching between the two groups was mainly due to the ideal LA matching rather than the ideal IP matching. Changing the original IP of the spine can easily lead to overcorrection of spinal deformities, thus increasing the stress on the internal fixation system and then the risk of mechanical complications. Therefore, it is more important to adjust LA of ADS patients during surgery. Our study showed: there was no significant correlation between 
Roussouly-type matching and mechanical complications; the ROC analysis implied that Roussouly-type matching could not accurately predict the risk of mechanical complications. Roussouly-type only morphologically described the sagittal characteristics of ADS patients, which lacked three-dimensional analysis and quantitative indicators of the spinal deformity in ADS patients. Therefore, the usage of Roussouly classification in the realignment of ADS is difficult.

In contrast, the GAP score quantifies the imaging parameters as well as the age of the patients, thus intuitively predicting the risk of postoperative mechanical complications in ADS patients [1]. However, there is no study comparing Roussouly classification with GAP score in their effectiveness of predicting the risk of mechanical complications following ADS surgery. In our study, GAP was better than Roussouly classification in predicting mechanical complications; GAP score was mostly effective in predicting PJK and PJF; however, the prediction accuracy of GAP for implant breakage and DJK or DJF is low. This is because implant breakage is closely related to the material properties of the internal fixation system itself, the living habits of patients and the overall structure of the internal fixation. The occurrence of DJK is affected by many factors, such as the distal fixation method, the severity of ADS and the levels of internal fixation; these factors are not fully reflected in GAP score, so the accuracy of prediction is also low [22].

There are some limitations in this study. First, because older patients are more sensitive to spinal sagittal orthodontics, the patients included in this study were older than those in previous studies. This may make the results of this study different from those of previous studies. However, the age span of patients included in this study was small, so the conclusion of this study is more accurate when applied to elderly patients. Second, this study only analyzed the parameters involved in Roussouly classification and GAP score, while did not discuss the conditions of paraspinal muscles and lower limb compensations. This will prevent the results of this study from explaining all the causes of postoperative mechanical complications. For example, if the paravertebral muscles of the thoracic vertebrae are weak and the PT is large (pelvic compensation is poor), the compensatory capability of the patient without lower limb compensation will be poor; then the sagittal imbalance of the spine will develop quickly. Despite the deficiencies mentioned above, this study compared the Roussouly classification with the GAP score through careful statistical analysis, and the results of this study were still of high reference value. A new classification method, considering the compensation of patient-specific spinal alignment and spinal balance, is still needed.

\section{Conclusion}

In matching Roussouly-type during surgical treatment for elderly ADS patients, changing the original IP of the spine can easily lead to overcorrection of spinal deformities, thus increasing the stress on the internal fixation system and then the risk of mechanical complications. It is more important to adjust LA of ADS patients during surgery. Roussouly-type matching could not accurately predict the risk of mechanical complications. In contrast, GAP score was mostly effective in predicting PJK and PJF. The GAP score was better than Roussouly classification in predicting mechanical complications. 


\section{Abbreviations}

ADS = adult degenerative scoliosis, $\mathrm{SRS}=$ Scoliosis Research Society, GAP = Global Alignment and Proportion, $\mathrm{PI}=$ pelvic incidence, $\mathrm{LL}=$ lumbar lordosis, $\mathrm{PT}$ = pelvic tilt, $\mathrm{SVA}=$ sagittal vertical axis, $\mathrm{SS}=$ sacral slope, VAS = visual analogue scale, JOA = Japanese Orthopaedic Association, ODI = Oswestry Disability Index, CA = coronal Cobb angle, TK = thoracic kyphosis, TLK = thoracolumbar kyphosis, GT = global tilt, NVL = the number of vertebrae included in the lordosis, $L A=$ lumbar sagittal apex, IP = inflexion point, $\mathrm{RPV}=$ relative pelvic version, $\mathrm{PLL}=$ relative lumbar lordosis, $\mathrm{LDI}=$ lordosis distribution index, $\mathrm{RSA}=$ relative spinopelvic alignment, $\mathrm{PJK}=$ proximal junctional kyphosis, $\mathrm{PJF}=$ proximal junctional failure, $\mathrm{DJK}$ = distal junctional kyphosis, DJF = distal junctional failure, UIV = upper instrumented vertebra, LIV = lower instrumented vertebra, $\mathrm{OR}=$ odds ratio, $\mathrm{Cl}=$ confidence interval, $\mathrm{ROC}=$ receiver operating characteristic.

\section{Declarations}

\section{Acknowledgments}

The authors would like to thank Professor Shibao Lu and Dr. Chao Kong who provided data support and corrected some mistakes. They also would like to thank Xiangyao Sun M.D., Ph.D, for innovative thinking and thesis writing.

\section{Compliance with ethical standards}

\section{Conflict of interest}

The authors declared that they had no conflict of interest.

\section{Funding}

This study was financially supported by China Scholarship Council (No.201908110337), National Natural Science Foundation of China (No. 81672201, No. 81871794) and Beijing Municipal Commission of Health and Family Planning (No. PXM2017 026283 000002).

\section{Ethical approval}

The experimental protocol was established according to the ethical guidelines of the Helsinki Declaration and was approved by the Human Ethics Committee of Xuanwu Hospital Capital Medical University (No. Lin Yan Shen [2018] 014).

\section{Informed consent}

Informed consents were obtained from individual participants included in the study.

\section{References}


1. Pizones J, Moreno-Manzanaro L, Sanchez PF, Vila-Casademunt A, Yilgor C, Obeid I, Alanay A, Kleinstuck F, Acaroglu ER, Pellise F. Restoring the ideal Roussouly sagittal profile in adult scoliosis surgery decreases the risk of mechanical complications. EUR SPINE J. 2020; 29(1): 54-62. https://doi.org/10.1007/s00586-019-06176-x.

2. Barrey C, Jund J, Noseda O, Roussouly P. Sagittal balance of the pelvis-spine complex and lumbar degenerative diseases. A comparative study about 85 cases. EUR SPINE J. 2007; 16(9): 1459-1467. https://doi.org/10.1007/s00586-006-0294-6.

3. Schwab F, Patel A, Ungar B, Farcy JP, Lafage V. Adult spinal deformity-postoperative standing imbalance: how much can you tolerate? An overview of key parameters in assessing alignment and planning corrective surgery. Spine (Phila Pa 1976). 2010; 35(25): 2224-2231. https://doi.org/10.1097/BRS.0b013e3181ee6bd4.

4. Roussouly P, Gollogly S, Berthonnaud E, Dimnet J. Classification of the normal variation in the sagittal alignment of the human lumbar spine and pelvis in the standing position. Spine (Phila $\mathrm{Pa}$ 1976). 2005; 30(3): 346-353. https://doi.org/10.1097/01.brs.0000152379.54463.65.

5. Yilgor C, Sogunmez N, Boissiere L, Yavuz Y, Obeid I, Kleinstuck F, Perez-Grueso F, Acaroglu E, Haddad S, Mannion AF, et al. Global Alignment and Proportion (GAP) Score: Development and Validation of a New Method of Analyzing Spinopelvic Alignment to Predict Mechanical Complications After Adult Spinal Deformity Surgery. J BONE JOINT SURG AM. 2017; 99(19): 1661-1672. https://doi.org/10.2106/JBJS.16.01594.

6. Schwab F, Ungar B, Blondel B, Buchowski J, Coe J, Deinlein D, DeWald C, Mehdian H, Shaffrey C, Tribus $\mathrm{C}$, et al. Scoliosis Research Society-Schwab adult spinal deformity classification: a validation study. Spine (Phila Pa 1976). 2012; 37(12): 1077-1082. https://doi.org/10.1097/BRS.0b013e31823e15e2.

7. Soroceanu A, Diebo BG, Burton D, Smith JS, Deviren V, Shaffrey C, Kim HJ, Mundis G, Ames C, Errico T, et al. Radiographical and Implant-Related Complications in Adult Spinal Deformity Surgery: Incidence, Patient Risk Factors, and Impact on Health-Related Quality of Life. Spine (Phila Pa 1976). 2015; 40(18): 1414-1421. https://doi.org/10.1097/BRS.0000000000001020.

8. Laouissat F, Sebaaly A, Gehrchen M, Roussouly P. Classification of normal sagittal spine alignment: refounding the Roussouly classification. EUR SPINE J. 2018; 27(8): 2002-2011. https://doi.org/10.1007/s00586-017-5111-x.

9. Sebaaly A, Grobost P, Mallam L, Roussouly P. Description of the sagittal alignment of the degenerative human spine. EUR SPINE J. 2018; 27(2): 489-496. https://doi.org/10.1007/s00586-0175404-0.

10. Barrey C, Roussouly P, Perrin G, Le Huec JC. Sagittal balance disorders in severe degenerative spine. Can we identify the compensatory mechanisms? EUR SPINE J. 2011; 20 Suppl 5: 626-633. https://doi.org/10.1007/s00586-011-1930-3.

11. Yilgor C, Sogunmez N, Yavuz Y, Abul K, Boissiere L, Haddad S, Obeid I, Kleinstuck F, Sanchez PF, Acaroglu E, et al. Relative lumbar lordosis and lordosis distribution index: individualized pelvic 
incidence-based proportional parameters that quantify lumbar lordosis more precisely than the concept of pelvic incidence minus lumbar lordosis. NEUROSURG FOCUS. 2017; 43(6): E5. https://doi.org/10.3171/2017.8.FOCUS17498.

12. Pizones J, Martin MB, Perez-Grueso F, Yilgor C, Vila-Casademunt A, Serra-Burriel M, Obeid I, Alanay A, Acaroglu ER, Pellise F. Impact of Adult Scoliosis on Roussouly Sagittal Shape Classification. Spine (Phila Pa 1976). 2019; 44(4): 270-279. https://doi.org/10.1097/BRS.0000000000002800.

13. Berthonnaud E, Dimnet J, Roussouly P, Labelle H. Analysis of the sagittal balance of the spine and pelvis using shape and orientation parameters. J SPINAL DISORD TECH. 2005; 18(1): 40-47. https://doi.org/10.1097/01.bsd.0000117542.88865.77.

14. Schwab FJ, Hawkinson N, Lafage V, Smith JS, Hart R, Mundis G, Burton DC, Line B, Akbarnia B, Boachie-Adjei O, et al. Risk factors for major peri-operative complications in adult spinal deformity surgery: a multi-center review of 953 consecutive patients. EUR SPINE J. 2012; 21(12): 2603-2610. https://doi.org/10.1007/s00586-012-2370-4.

15. Bianco K, Norton R, Schwab F, Smith JS, Klineberg E, Obeid I, Mundis GJ, Shaffrey Cl, Kebaish K, Hostin R et al. Complications and intercenter variability of three-column osteotomies for spinal deformity surgery: a retrospective review of 423 patients. NEUROSURG FOCUS. 2014; 36(5): E18. https://doi.org/10.3171/2014.2.FOCUS1422.

16. Hallager DW, Karstensen S, Bukhari N, Gehrchen M, Dahl B. Radiographic Predictors for Mechanical Failure After Adult Spinal Deformity Surgery: A Retrospective Cohort Study in 138 Patients. Spine (Phila Pa 1976). 2017; 42(14): E855-E863. https://doi.org/10.1097/BRS.0000000000001996.

17. Boissiere L, Takemoto M, Bourghli A, Vital JM, Pellise F, Alanay A, Yilgor C, Acaroglu E, Perez-Grueso FJ, Kleinstuck F, et al. Global tilt and lumbar lordosis index: two parameters correlating with healthrelated quality of life scores-but how do they truly impact disability? SPINE J. 2017; 17(4): 480-488. https://doi.org/10.1016/j.spinee.2016.10.013.

18. Obeid I, Boissiere L, Yilgor C, Larrieu D, Pellise F, Alanay A, Acaroglu E, Perez-Grueso FJ, Kleinstuck F, Vital JM, et al. Global tilt: a single parameter incorporating spinal and pelvic sagittal parameters and least affected by patient positioning. EUR SPINE J. 2016; 25(11): 3644-3649.

https://doi.org/10.1007/s00586-016-4649-3.

19. Inoue S, Khashan M, Fujimori T, Berven SH. Analysis of mechanical failure associated with reoperation in spinal fusion to the sacrum in adult spinal deformity. J ORTHOP SCI. 2015; 20(4): 609616. https://doi.org/10.1007/s00776-015-0729-1.

20. Jacobs E, van Royen BJ, van Kuijk S, Merk J, Stadhouder A, van Rhijn LW, Willems PC. Prediction of mechanical complications in adult spinal deformity surgery-the GAP score versus the Schwab classification. SPINE J. 2019; 19(5): 781-788. https://doi.org/10.1016/j.spinee.2018.11.013.

21. Schwab FJ, Blondel B, Bess S, Hostin R, Shaffrey Cl, Smith JS, Boachie-Adjei O, Burton DC, Akbarnia BA, Mundis GM, et al. Radiographical spinopelvic parameters and disability in the setting of adult spinal deformity: a prospective multicenter analysis. Spine (Phila Pa 1976). 2013; 38(13): E803E812. https://doi.org/10.1097/BRS.0b013e318292b7b9. 
22. Bari TJ, Hansen LV, Gehrchen M. Surgical correction of Adult Spinal Deformity in accordance to the Roussouly classification: effect on postoperative mechanical complications. Spine Deform. 2020; 8(5): 1027-1037. https://doi.org/10.1007/s43390-020-00112-6.

23. Roussouly P, Nnadi C. Sagittal plane deformity: an overview of interpretation and management. EUR SPINE J. 2010; 19(11): 1824-1836. https://doi.org/10.1007/s00586-010-1476-9

24. Legaye J, Duval-Beaupere G, Hecquet J, Marty C. Pelvic incidence: a fundamental pelvic parameter for three-dimensional regulation of spinal sagittal curves. EUR SPINE J. 1998; 7(2): 99-103. https://doi.org/10.1007/s005860050038.

25. Sebaaly A, Gehrchen M, Silvestre C, Kharrat K, Bari TJ, Kreichati G, Rizkallah M, Roussouly P. Mechanical complications in adult spinal deformity and the effect of restoring the spinal shapes according to the Roussouly classification: a multicentric study. EUR SPINE J. 2020; 29(4): 904-913. https://doi.org/10.1007/s00586-019-06253-1.

26. Bae J, Lee SH, Shin SH, Seo JS, Kim KH, Jang JS. Radiological analysis of upper lumbar disc herniation and spinopelvic sagittal alignment. EUR SPINE J. 2016; 25(5): 1382-1388. https://doi.org/10.1007/s00586-016-4382-y.

\section{Figures}




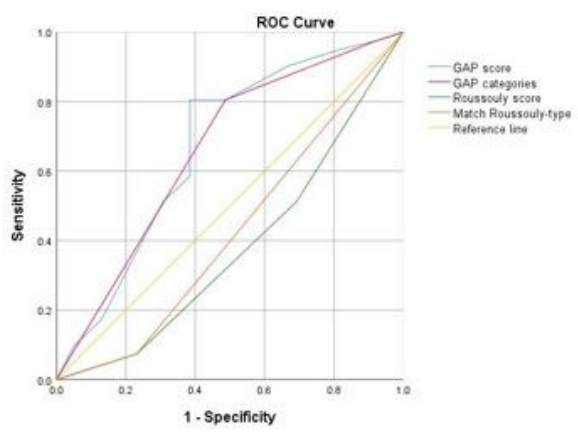

(a) Mechanical complications

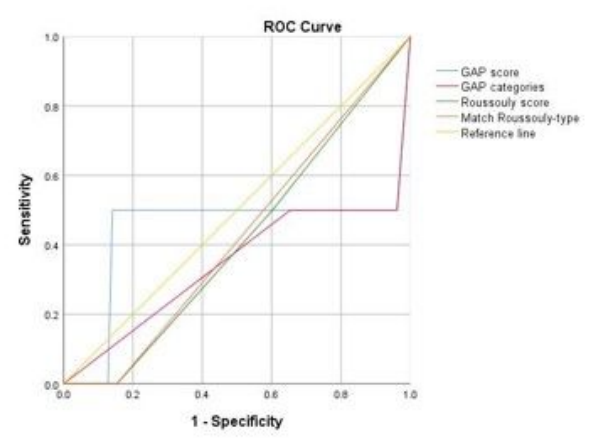

(c) PJF

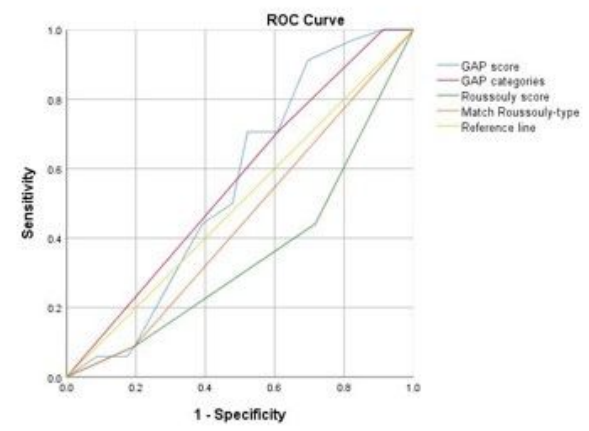

(e) Implant-related complications

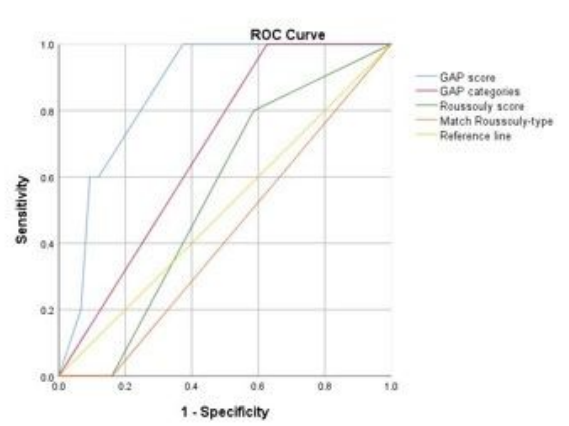

(b) PJK

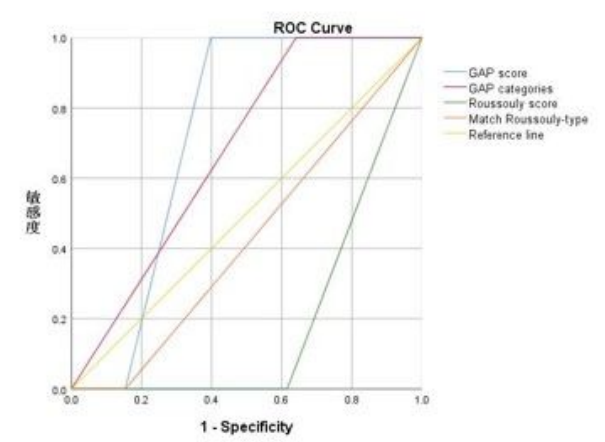

(d) DJF or DJF

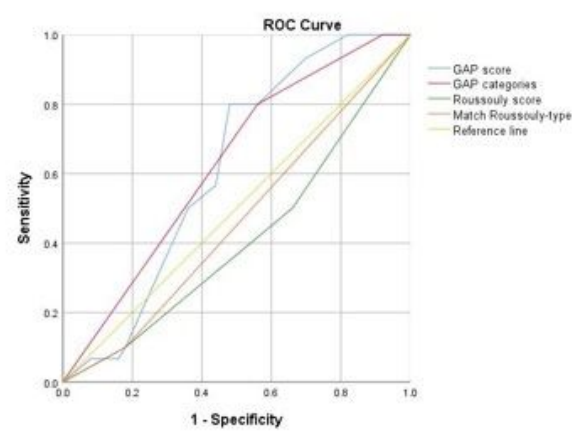

(f) Implant loosening

\section{Figure 1}

ROC curve of evaluation systems in predicting (a) Mechanical complications, (b) PJK, (c) PJF, (d) DJK or DJF, (e) Implant-related complications and (f) Implant loosening.

\section{Supplementary Files}


This is a list of supplementary files associated with this preprint. Click to download.

- Suppelmentaryfile3.docx

- Supplementaryfile1.docx

- Supplementaryfile2.docx 